\title{
Untersuchungen zum Vorkommen von Myxobakterien in von Meerwasser beeinflußten Substraten unter besonderer Berücksichtigung der Insel Helgoland
}

\author{
G. Rückert
}

Botanisches Institut I und Botanischer Garten der Universität (TH); D-7500 Karlsruhe, Bundesrepublik Deutschland

\begin{abstract}
Investigations on the distribution of myxobacteria in substrates influenced by seawater with special reference to the island of Helgoland. Representatives of the family Myxococcaceae, Myxococcus fulvus and $M$. virescens as well as Archangium gephyra could be isolated from marine sediments (depth range 5-58 m), collected near the island of Helgoland (North Sea); dunes and rudiments of salt marshes additionally yielded $M$. coralloides and the rare species Melittangium lichenicola and $M$. boletus (Cystobacteriaceae). In soil samples from the island, $M$. fulvus, $M$. virescens, $M$. coralloides, A. gephyra, Cystobacter fuscus and Stigmatella erecta were found. These results were confirmed by data, obtained from the coastal zone of the island of Amrum and marine sediments from various regions. On the other hand samples from shallow fresh water (depth range $0.3-1 \mathrm{~m}$ ) proved to be richer in species. It is assumed that the myxobacteria found in marine sediments occur as resting cells.
\end{abstract}

\section{EINLEITUNG}

Vertreter der Bakterienordnung Myxobacterales gelten als typische Organismen der Boden-Mikroflora und sind dort in Abhängigkeit natürlicher und wirtschaftsbedingter Bodenentwicklungen in wechselnder Arten-Zusammensetzung anzutreffen (Peterson, 1969; Rückert, 1979; Reichenbach \& Dworkin, 1981). Darüber hinaus gibt es zahlreiche Hinweise auf weitere terrestrische Vorkommen wie in Mist von Pflanzenfressern (Jahn, 1924; Rückert, 1972), auf Borke (Peterson, 1957; Dawid, 1977) oder in Substraten, die extremen ökologischen Bedingungen unterliegen, wie in Hochmooren (Dawid, 1983) oder in Vollwüsten extrem arider Gebiete (Rückert, 1983).

Demgegenüber sind Erhebungen über die Myxobakterien-Flora der Gewässer oder subhydrischer Sedimente selten und erst in neuerer Zeit systematisch durchgeführt worden, denn es dauerte nach der Beschreibung von Polyangium parasiticum als Cladophora-Parasit (Geitler, 1924) Jahrzehnte, bis sich die Aufmerksamkeit der Bakteriologen erneut dem Biotop Süßwasser zuwandte und in einigen unabhängig voneinander durchgeführten Isolierungsversuchen sowohl aus stehenden als auch aus Fließgewässern im wesentlichen Vertreter der Gattung Myxococcus nachgewiesen werden konnten (Brockman, 1971; Raverdy, 1973; Gräf, 1975).

Ganz anders verhält es sich mit freiem Meerwasser: Hier gibt es erst einen Hinweis darauf, daß Myxokokken, wenn auch selten, vorhanden sind (Brockman, 1973). Dagegen 
finden sich weltweit in größerer Anzahl Hinweise auf die Strand- und Dünenregionen, die sehr reich an verschiedenen Gattungen sein können (Brockman, 1970; Rückert, 1975).

Deshalb erscheint es etwas überraschend, daß Sedimente sowohl unter Süß- als auch unter Meerwasser bisher unberücksichtigt blieben. Drei Aufenthalte des Verfassers auf Helgoland in den Jahren 1982 und 1983 erwiesen sich als gute Gelegenheit, neben Proben aus Salzwiesen-Rudimenten, Spülsäumen und Weißdünen auch marine Sedimente zu erfassen. Die isolierte Lage der Insel - bei aller Gefahr und Wertung eines bakteriologischen Einflusses touristischer Masseninfektionen, die bei Myxobakterien allerdings relativ gering ausfallen - bietet zudem die Möglichkeit, einen Vergleich mit den Befunden anzustreben, wie sie aus landbürtigen Böden vorliegen. Die unvollständige Strand-Dünen-Abfolge Helgolands ließ es außerdem angebracht erscheinen, kurz auf die Verhältnisse auf Amrum einzugehen und bereits vorliegende Daten aus Sedimentuntersuchungen miteinzubeziehen (Rückert, 1980).

\section{MATERIAL UND METHODEN}

Eine Besonderheit der Myxobakterien ist ihre Fähigkeit, Fruchtkörper verschiedener Größe, Form und Färbung zu bilden, die in der Regel eine Ansprache der Arten in Rohkulturen nach 10 - bis 20 tägiger Bebrütung bei $30^{\circ} \mathrm{C}$ mit dem Binokular bei 10 - bis 30facher Vergrößerung zulassen (Abbildungen u. a. bei McCurdy, 1969; Reichenbach, 1974; Reichenbach \& Dworkin, 1981). Hierzu wurden von uns die folgenden zwei Grundmethoden angewandt:

(1) Auf 10 neutrale Wasseragar-Platten wurden je $2 \mathrm{mg}$ in Leitungswasser autoklavierte Bierhefezellen ausgestrichen und nach dem Antrocknen pro Platte 5 Probenhäufchen ausgebracht (modifiziert nach Singh, 1947).

(2) 5 Petrischalen wurden mit je 1 gehäuften Eßlöffel Probenmaterials beschickt und nach guter Durchfeuchtung je 10 autoklavierte Wildhasen- oder Wildkaninchen-Mistköder zur Hälfte in das Material eingedrückt (modifiziert nach Krzemieniewska \& Krzemieniewski, 1926).

Geringe Kochsalzzugaben in den Agar $(0,5 \%)$ bzw. in der Lösung zur erstmaligen Durchfeuchtung der Mistplatten (1\%) fördern bei salzfreien und salzarmen Substraten die Fruchtkörper-Bildung von Myxococcus virescens (Rückert, 1978). Wo immer diese Art fehlte, wurden die Methoden nach (1) und (2) mit je 25 Einzelansätzen unter NaClZugabe ergänzt.

Das gesiebte und luftgetrocknete Untersuchungsmaterial setzte sich entsprechend der nachfolgenden Angaben zusammen.

(1) Helgoland: terrestrische Proben 9, Weißdünen 3, Spülsäume 9, SalzwiesenRudimente 2, submerse Proben bis $-5 \mathrm{~m} 3 \mathrm{bzw}$, um $-50 \mathrm{~m} 4$ (.,Tiefe Rinne ");

(2) Amrum: Dünenwald 1, Tertiärdünen 3, Weißdünen 4, Vordünen 4 und Spülsäume 4.

(3) Submerse Proben verschiedener Herkunft (Rückert, 1980) 81

Die pH-Werte der Proben wurden elektrometrisch als $\mathrm{pH}_{\left(\mathrm{H}_{2} \mathrm{O}\right)}(1: 2,5)$ ermittelt.

Die Nomenklatur der Familien, Gattungen und Arten richtet sich nach McCurdy (1974). 


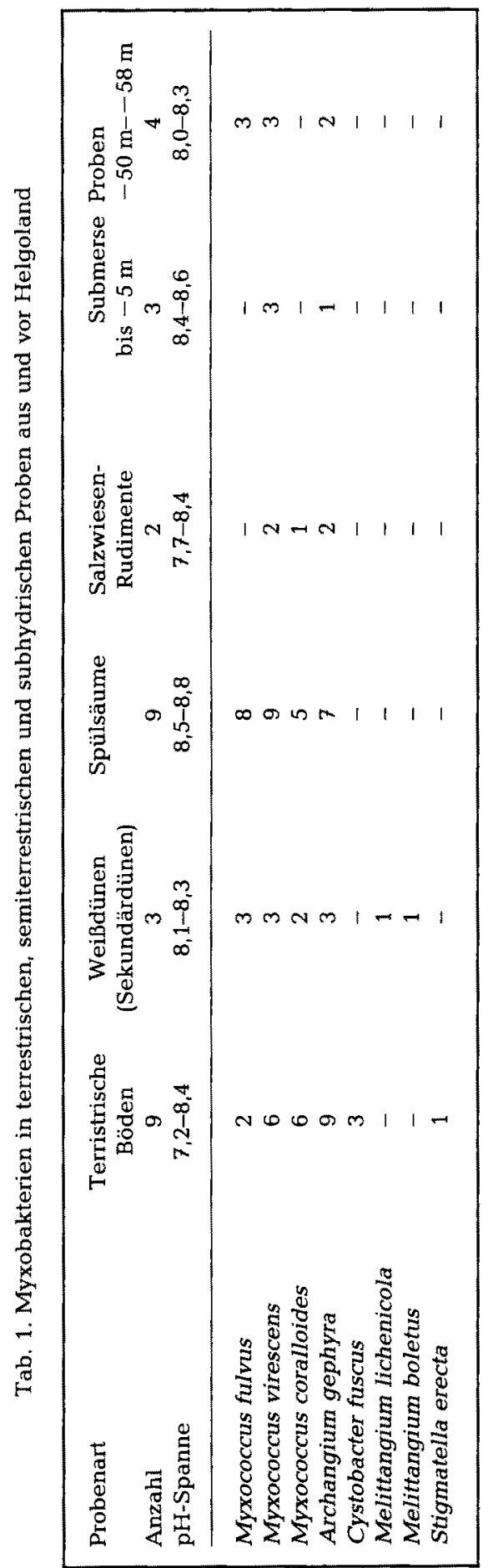


Tab. 2. Myxobakterien in Spülsaum-Dünen-Abfolgen vom westlichen Amrum

\begin{tabular}{|lccccc|}
\hline Probenart & Spülsaum & Primärdüne & Sekundärdüne & Tertiärdüne & Dünenwald \\
Anzahl & 4 & 4 & 4 & 3 & 1 \\
pH-Spanne & $6,5-8,0$ & $7,0-8,7$ & $5,5-8,1$ & $4,4-5,6$ & 4,1 \\
\hline Myxococcus fulvus & 2 & 3 & 4 & 3 & 1 \\
Myxococcus virescens & 4 & 3 & 4 & 3 & - \\
Myxococcus coralloides & 3 & 4 & 4 & 3 & 1 \\
Archangium gephyra & 1 & 4 & 3 & 2 & - \\
Cystobacter fuscus & - & - & 1 & - & - \\
Melittangium boletus & - & - & 2 & 1 & - \\
\hline
\end{tabular}

\section{ERGEBNISSE}

Die in Tabelle 1 dargelegten Befunde zeigen trotz der relativ geringen Probenzahl aus Helgoland mit 8 nachgewiesenen Arten eine bemerkenswerte Vielfalt, zumal auf die Bearbeitung von Gartenböden verzichtet wurde. Stigmatella erecta und die beiden Melittangium-Arten gelten allgemein als selten. Vor allem $M$. boletus ist bis heute nur ganz vereinzelt gefunden worden; die Art scheint tatsächlich einen Verbreitungsschwerpunkt in Dünen aufzuweisen. Hervorzuheben ist auch die Arten-Diskrepanz zwischen subhydrischen, semiterrestrischen und terrestrischen Proben. Die Familie der Cystobacteriaceae mit den Gattungen Cystobacter, Stigmatella und Melittangium erhält Differentialcharakter: Sie fehlt auch unter Miteinbeziehung der zusätzlichen Daten in den marinen Sedimenten und im Spülsaumbereich völlig, und dies selbst nach gelegentlich durchgeführter Entsalzung der Proben. So wird auch in Tabelle 2 mit Proben vom westlichen Teil Amrums die Tendenz erhärtet, daß die Gattungen Myxococcus und Archangium in solchen Proben dominieren. Sie erhält dadurch besonderes Gewicht, daß diese Proben in Serien vom Strand zum Inselinneren entnommen wurden; dies wird vor allem auch im Vergleich zwischen Spülsäumen und Vordünen von Rückert (1975) und durch einige Probenserien aus Sylt und dem westlichen Dänemark bestätigt.

Tab. 3. Myxobakterien in submersen Proben unterschiedlicher Herkunft, nach dem Gewässertyp bzw, der Entnahmetiefe geordnet

\begin{tabular}{|c|c|c|c|c|}
\hline Gewässertyp & $\begin{array}{l}\text { Süßwasser } \\
\text { (fließend) }\end{array}$ & $\begin{array}{l}\text { Süßwasser } \\
\text { (stehend) }\end{array}$ & Meerwasser & Meerwasser \\
\hline Entnahmetiefe (m) & $0,3-1$ & $0,3-1$ & $0,5-4$ & $5-50$ \\
\hline pH-Spanne & $4,2-8,6$ & $5,0-8,7$ & $8,2-9,3$ & $7,4-9,3$ \\
\hline Anzahl & 30 & 23 & 13 & 15 \\
\hline ohne Befund & 1 & - & 2 & 3 \\
\hline Myxococcus fulvus & 18 & 12 & 5 & 1 \\
\hline Myxococcus virescens & 23 & 17 & 8 & 11 \\
\hline Myxococcus coralloides & 22 & 19 & 2 & 4 \\
\hline Archangium gephyra & 22 & 12 & 2 & 2 \\
\hline Cystobacter fuscus & 1 & - & - & - \\
\hline Chondromyces crocatus & - & 1 & - & - \\
\hline Polyangium sorediatum & 2 & 1 & - & - \\
\hline
\end{tabular}


Die Verarmung subhydrischer Sedimente gilt in diesem Umfang für Proben unter stehenden und fließenden flachen Süßgewässern nicht, wo neben Myxococcaceen und Archangium gephyra auch zusätzliche Taxa auftreten (Tab. 3). Unter den MyxococcusArten ist der starke Rückgang von $M$. coralloides unter marinen Bedingungen bemerkenswert.

\section{DISKUSSION}

Bei allen Erörterungen über die Verbreitung von Mikroorganismen darf nie die Frage außer acht bleiben, inwiefern Artennachweise daraus resultieren, daß man sich teilende, also aktive, oder nur ruhende teilungsfähige, also potentiell aktive Stadien vorfindet. Fruchtkörper-Bildung unter optimalen Laborbedingungen kann sowohl aus intakten Ruhestadien als auch aus aktiven Stadien aus dem Substrat heraus erfolgen. Eine Infektion jedweden Substrats mit Mikroorganismen ist indessen $u$. a. durch Wind, Wasser, Bodeneintrag jederzeit möglich. Myxobakterien sind heterotrophe mesophile Aerobier mit überwiegend proteolytischer, z. T. auch cellulolytischer Aktivität. Wachstum bei Temperaturen unter $15^{\circ} \mathrm{C}$ wurde bislang nicht beobachtet (McCurdy, 1974), wohl aber ein Überdauerungsvermögen der kürzeren und derberen Ruhezellen von sechs (Reichenbach, 1974) bis über zehn Jahren (Rückert, noch nicht veröffentlicht) in lufttrockenen Bodenproben bei Zimmertemperatur. Auch aus diesem Zustand heraus ist eine Fruchtkörper-Bildung binnen weniger Tage möglich, so daß zumindest das Substrat „Wüstenboden" durchaus eine eigene echte Myxobakterien-Flora aufweisen kann. Unwahrscheinlich, wenn nach heutigen Kenntnissen nicht sogar unmöglich, erscheint uns aber der Biotop „Wasser" oder „Sediment unter Wasser" zu sein, sofern andauernd weitgehend anaerobe und/oder zu kühle Bedingungen vorliegen.

Empfindlich reagieren viele Myxobakterien-Arten bei $\mathrm{NaCl}$-Gehalten im Substrat über $0,5 \%$, was ihre Fähigkeit zur Fruchtkörper-Bildung und damit die Identifizierungsmöglichkeit anlangt. Hier ist vor allem Myxococcus virescens eine Ausnahme: Vereinzelt konnte bei dieser Art noch eine Fruchtkörper-Bildung bei Zugabe von $4 \%$ Meersalz in ein salzfreies Substrat beobachtet werden. Ihre Häufigkeit in saliner Umgebung ist deshalb kein Zufall.

Die starke relative Zunahme von Myxococcaceen und Archangium gephyra in Sedimenten unter Salzwasser sehen wir im wesentlichen darin begründet, daß ihre im Vergleich mit den vegetativen Stäbchen besonders kurzen und derben Ruhestadien überdurchschnittlich widerstandsfähig sind. Wir folgen hier der Vermutung Brockmans (1973), wollen dies aber für Süßwasser im Gegensatz zu Angerstein (1973) wesentlich differenzierter sehen: Hier scheinen zumindest periodisch, in tropischen Gewässern unter Umständen ständig, günstige Entwicklungsmöglichkeiten für Myxobakterien zu bestehen, wie es u. a. von Raverdy (1973) und besonders von Gräf (1975) gefordert wird.

In einer kritischen Wertung der für Myxobakterien von Art zu Art unterschiedlich wichtigen ökologischen Parameter, Sauerstoff- und Wärmebedarf, i. a. geringe Belastbarkeit durch Salze und niedere pH-Werte, Verfügbarkeit lysierbarer Makromoleküle oder niedermolekularer, direkt verwertbarer Nahrung, in Verbindung mit ihrer Fähigkeit zur Bildung von Dauerstadien, erscheinen die Daten aus Helgoland als eindrucksvoller Beweis, einzelne Bakterienarten mit Maßen zur Kennzeichnung von Biotopen heranzuziehen. 
Danksagungen. Den Besatzungen der Forschungskutter "Uthörn" danke ich für die Beschaffung der Proben aus der "Tiefen Rinne“ vor Helgoland in den Jahren 1982 und 1983, meiner Mitarbeiterin Frau U. Hütz für die zuverlässige Erledigung der praktischen Laborarbeiten.

\section{ZITIERTE LITERATUR}

Angerstein, H, 1973. Uber das Vorkommen von fruchtkörperbildenden Myxobakterien in der Uferzone des Bodensees. Staatsexamensarb., Karlsruhe, $34 \mathrm{pp}$.

Brockman, E. R., 1970. Isolation and distribution of fruiting myxobacteria in Atlantic coast beaches. - Bact. Proc. 1970, 27.

Brockman, E. R., 1971. Fruiting myxobacteria in Michigan lakes and streams. - Bact. Proc. 1971, 52.

Brockman, E. R., 1973. Isolation of myxobacteria from marine habitats in the U. S. Virgin Islands. In: Estuarine microbial ecology. Ed. by L. H. Stevenson \& R. R. Colwell. Univ. of South Carolina Press, Columbia, S. C., 1, 45-52.

Dawid, W., 1977. Vorkommen und Verbreitung fruchtkörperbildender Myxobakterien im Siebengebirge. Diss., Bonn, $96 \mathrm{pp}$.

Dawid, W., 1983. Zur Mikroflora der Hochmoore im Hohen Venn (Belgien) unter besonderer Berücksichtigung der Myxobakterien. - Telma 13, 161-171.

Geitler, L., 1924, Über Polyangium parasiticum n. sp., eine submerse, parasitische Myxobacteriacee. - Arch. Protistenk. 50,67-88.

Gräf, W., 1975. Myxobakterien der Gattung Myxococcus als indirekte Fäkalstoffindikatoren in Oberflächengewässern. 1. Mitteilung - Zbl. Bakt. Parasitkde. (Abt. 1B) 160, 28-38.

Jahn, E., 1924. Beiträge zur botanischen Protistologie. I. Die Polyangiden. Borntraeger, Leipzig, $107 \mathrm{pp}$.

Krzemieniewska, H. \& Krzemieniewski, S., 1926. Die Myxobakterien von Polen. - Acta Soc. Bot. Pol. 4, 1-54.

McCurdy, H. D., 1969. Studies on the taxonomy of the Myxobacterales. I. Record of Canadian isolates and survey of methods. - Can. J. Microbiol. 15, 1453-1461.

McCurdy, H. D. 1974. Myxobacterales. The fruiting myxobacteria. In: Bergey's manual of determinative bacteriology. Ed. by R. E. Buchanan \& N. E. Gibbons. Williams \& Wilkins, Baltimore, $76-98$.

Peterson, J. E., 1957. The bark of living trees as a substrate for representatives of the order Myxobacteriales. Univ. Microfilms, Ann Arbor, $157 \mathrm{pp.}$

Peterson, J. E., 1969. The fruiting myxobacteria: their properties, distribution and isolation. J. appl. Bact. 32, 5-12.

Raverdy, J., 1973. Sur l'isolement et l'activité bacteriolytique de quelques myxobacteries isolées de l'eau. - Wat. Res. 7, 687-693.

Reichenbach, H., 1974. Die Biologie der Myxobakterien. - Biologie unserer Zeit 4, 33-45.

Reichenbach, H. \& Dworkin, M., 1981. The order Myxobacterales. In: The prokaryotes. Ed. by M. P. Starr, H. Stolp, H. G. Trüper, A. Balows, H. G. Schlegel. Springer, Berlin, 328-355.

Rückert, G., 1972. Vergleichende Untersuchungen über die Verbreitung einiger fruchtkörperbildender Myxobakterien-Arten. - Z. allg. Mikrobiol. 12, 655-665.

Rückert, G., 1975. Zur Verbreitung von fruchtkörperbildenden Myxobakterien in europäischen Strand- und Dünenböden. - Zbl. Bakt. Parasitkde. (Abt. 2) 130, 343-347.

Rückert, G., 1978. Förderung der Fruchtkörper-Bildung von Myxococcus virescens THAXTER (Myxobacterales) in Rohkulturen durch Salzzusatz. $-Z$. allg. Mikrobiol. 18, 69-71.

Rückert, G., 1979. Myxobakterien-Artenspektren von Böden in Abhängigkeit von bodenbildenden Faktoren unter besonderer Berücksichtigung der Bodenreaktion. $-Z$. Pfl. Ernähr. Düng. Bodenk. $142,330-343$.

Rückert, G, 1980. Beiträge zur Verbreitung, Verbreitungsökologie und Okologie der Myxobakterien (Myxobacterales). Habilitationsschr., Karlsruhe, $190 \mathrm{pp}$.

Rückert, G., 1983. Myxobakterien in Böden extremer Biotope. - Mitt. dt. bodenkdl. Ges. 38, 355-360.

Singh, B. N., 1947. Myxobacteria in soils and composts; their distribution, number and lytic action on bacteria. - J. gen. Microbiol. 1, 1-10. 